\title{
Quartic Interband Exciton Couplings in Pump-Probe Spectroscopy of Light Harvesting Complexes ${ }^{\dagger}$
}

\author{
Darius Abramavicius and Shaul Mukamel* \\ Department of Chemistry, University of California, Irvine, California 92697-2025
}

Received: November 19, 2003; In Final Form: January 30, 2004

\begin{abstract}
The standard Frenkel exciton Hamiltonian for molecular aggregates only contains quadratic (intraband) couplings between chromophores. However, interband interactions between chromophores whose excitation frequences are well separated are dominated by quartic couplings. We show that the latter are responsible for spectral line shifts of the Carotenoids observed in pump-probe experiments of LH2 photosynthetic antenna following excitation of the chrolophylls (Herek; et al., Biochemistry 1998, 37, 7057).
\end{abstract}

\section{Introduction}

The interpretation of coherent multidimensional optical signals of coupled chromophores, and their inversion to yield structural and dynamical information, should be based on parametrized model Hamiltonians. ${ }^{1}$ Such Hamiltonians are commonly used in $\mathrm{NMR}^{2}$ but are less firmly established for electronic and vibrational chromophores.

Elementary excitations of molecular assemblies with nonoverlapping charge distributions (molecular crystals, ${ }^{3,4} \mathrm{~J}$ aggregates, ${ }^{5,6}$ organic nanostructures, ${ }^{7}$ and supramolecular structures $^{8-10}$ ) are known as Frenkel excitons..$^{3,11}$ These are delocalized collective excitations created by electrostatic intermolecular interactions. The exciton states may be directly observed in linear optical spectroscopy. A broad arsenal of nonlinear techniques, such as fluorescence depolarization, ${ }^{12}$ photon echo, ${ }^{13-16}$ hole burning, ${ }^{17-19}$ and pump-probe, ${ }^{20-24}$ provide additional information on the one-exciton and multiexciton state structure, relaxation, and migration. ${ }^{25}$

The Frenkel exciton Hamiltonian of an aggregate made out of two-level molecules is usually recast using the exciton creation and annihilation operators $\hat{B}_{m}^{\dagger} \equiv|m\rangle\langle 0|$ and $\hat{B}_{m} \equiv$ $|0\rangle\langle m|$, where $|0\rangle$ is the ground state and $|m\rangle$ is the excited state of the $m$ th chromophore with energy $\Omega_{m}{ }^{3}$

$$
\hat{H}_{0}=\sum_{m} \Omega_{m} \hat{B}_{m}^{\dagger} \hat{B}_{m}+\sum_{m n} J_{m n} \hat{B}_{m}^{\dagger} \hat{B}_{n}
$$

The first term represents the isolated chromophores, whereas the second constitutes quadratic intermolecular interactions resulting in resonant exciton transfer. Excitonic effects are controlled by the ratio $\kappa \equiv J / \Delta \Omega$, where $\Delta \Omega$ is a typical difference between excited-state energies of the various chromophores (sometimes denoted diagonal disorder). For $\kappa \gg 1$ the chromophores act cooperatively and excitations are delocalized. In the opposite limit, $\kappa \ll 1$, the chromophores are decoupled and excitonic effects are negligible. Static diagonal disorder as well as dynamic disorder arising from fluctuations of the environment reduce the degree of cooperativity.

In this paper we demonstrate that when $\kappa$ is small, additional, quartic, couplings, are important in third-order and higher spectroscopies, giving rise to interband excitonic effects. Application is made to pump-probe spectroscopy of the photo-

† Part of the special issue "Gerald Small Festschrift". synthetic antenna LH2 where spectral shifts were observed in the carotenoid band following excitations of the chlorophylls. ${ }^{20}$ The role of quartic couplings was recently discussed for vibrational excitons, ${ }^{26}$ where the $\kappa \ll 1$ regime can be engineered by isotopic substitutions.

\section{Generalized Frenkel Exciton Hamiltonian of Molecular Aggregates}

We consider a molecular aggregate made out of interacting multilevel chromophores, each with $(N+1)$ states (the ground state $+N$ excited states). Using this basis set, the electronic Hamiltonian is given by ${ }^{11}$

$$
\hat{H}_{e l}=\sum_{m} \sum_{a} \mathscr{H}_{m a} \rho_{m}^{a a}+\sum_{m n}^{m \neq n} \sum_{a b c d} \mathscr{H}_{m n}^{a b, c d} \rho_{m}^{a b} \rho_{n}^{c d}
$$

where $\rho_{m}^{a b} \equiv|m a\rangle\langle m b|$ is a complete basis set of operators of the $m$ th molecule ( $a=0, \ldots, N$ denotes the state of the molecule, 0 being the ground state) and $\mathscr{H}_{m a}$ and $\mathscr{H}_{m n}^{a b, c d}$ are matrix elements. The first term describes the individual molecules and the second represents intermolecular interactions.

The operators $\rho_{m}^{a b}$ can be recast using exciton creation $\hat{B}_{m a}^{\dagger} \equiv$ $|m a\rangle\langle m 0|$ and annihilation $\hat{B}_{m a} \equiv|m 0\rangle\langle m a|$ operators, where $a$ and $b=1, \ldots, N$. Using these definitions we get $\rho_{m}^{a b}=\hat{B}_{m a}^{\dagger} \hat{B}_{m b}$, $\rho_{m}^{a 0}=\hat{B}_{m a}^{\dagger}, \rho_{m}^{0 a}=\hat{B}_{m a}$, and $\rho_{m}^{00}=1-\sum_{a} \hat{B}_{m a}^{\dagger} \hat{B}_{m b}$. Substituting these in the Hamiltonian eq 2 gives ${ }^{27}$

$$
\hat{H}_{\mathrm{el}}=\hat{H}_{1}+\hat{H}_{2}+\hat{H}_{3}+\hat{H}_{4}
$$

where

$$
\begin{gathered}
\hat{H}_{1}=\sum_{m} \sum_{a} \Omega_{m a} \hat{B}_{m a}^{\dagger} \hat{B}_{m a} \\
\hat{H}_{2}=\sum_{m n}^{m \neq n}\left\{\sum_{a b} J_{m a, n b} \hat{B}_{m a}^{\dagger} \hat{B}_{n b}+\sum_{a b c d} K_{m, n}^{a b, c d} \hat{B}_{m a}^{\dagger} \hat{B}_{m c}^{\dagger} \hat{B}_{m b} \hat{B}_{n d}\right\} \\
\hat{H}_{3}=\sum_{m}\left\{\sum_{a}\left(V_{m a}^{\prime} \hat{B}_{m a}^{\dagger}+V_{m a}^{\prime \prime} \hat{B}_{m a}\right)+\sum_{a b}^{a \neq b} V_{m}^{\prime \prime \prime}{ }_{a b} \hat{B}_{m a}^{\dagger} \hat{B}_{m b}\right\}
\end{gathered}
$$




$$
\begin{aligned}
\hat{H}_{4}=\sum_{m n}^{m \neq n} \sum_{a b}\left(U_{m a, n b}^{\prime} \hat{B}_{m a}^{\dagger} \hat{B}_{n b}^{\dagger}+U_{m a, n b}^{\prime \prime} \hat{B}_{m a} \hat{B}_{n b}\right) \\
+\sum_{m n}^{m \neq n} \sum_{a b c}\left(W_{m, n}^{\prime a, b c} \hat{B}_{m a}^{\dagger} \hat{B}_{n b}^{\dagger} \hat{B}_{n c}+W_{m, n}^{\prime a b, c} \hat{B}_{m a}^{\dagger} \hat{B}_{m b} \hat{B}_{n c}\right)
\end{aligned}
$$

Equations 3-7 express the most general form of the exciton Hamiltonian in terms of the creation/annihilation operators. All coefficients $\Omega_{m a}, V_{m a}^{\prime}, V_{m a}^{\prime \prime}, V_{m, a b}^{\prime \prime \prime}, J_{m a, n b}, K_{m, n}^{a b, c d}, U_{m a, n b}^{\prime}, U_{m a, n b}^{\prime \prime}$, $W_{m, n}^{\prime a, b c}$, and $W_{m, n}^{\prime \prime} a b, c$ are linear combinations of $\mathscr{H}_{m a}^{m a}$ and $\mathscr{H}_{m n}^{b, c d}$. The various terms can be interpreted as follows: $\hat{H}_{1}$ represents the isolated molecule and $\hat{H}_{3}$ represents the contribution of the electric field on each chromophore created by other chromophores. This local field shifts the energy of the state $\left(\Omega_{m a}\right.$ $\left.\neq \mathscr{H}_{m a}\right)$ and couples the various energy levels through the terms $V_{m a}^{\prime}, V_{m a}^{\prime \prime}$, and $V_{m, a b}^{\prime \prime \prime} . \hat{H}_{3}$ is a single molecule coupling term that can be eliminated by rediagonalizing the single chromophore Hamiltonians. The coupling between chromophores is represented by $\hat{H}_{2}$ and $\hat{H}_{4} . \hat{H}_{2}$ has quadratic $J_{m a, n b}$ and quartic $K_{m, n}^{a b, c d}$ resonant couplings that conserve the number of excitons. $\hat{H}_{4}$ is off resonant and couples states with different numbers of excitons.

When the intermolecular coupling $\mathscr{H}_{m n}^{a b, c d}$ (typically smaller than $500 \mathrm{~cm}^{-1}$ in molecular aggregates) is much smaller than the transition energies $\mathscr{T}_{m a}\left(\sim 20000 \mathrm{~cm}^{-1}\right)$ the off-resonant terms given by $\hat{H}_{3}$ and $\hat{H}_{4}$ make small contributions to expectation values of the relevant observables and may be neglected.

The quartic terms can be recast in the form $\hat{B}_{m a}^{\dagger} \hat{B}_{n b}^{\dagger} \hat{B}_{m a} \hat{B}_{n b}$ $=\hat{B}_{m a}^{\dagger} \hat{B}_{m a} \hat{B}_{n b}^{\dagger} \hat{B}_{n b}$ (for $m \neq n$ ). The energy of a given exciton state is described by $\hat{B}_{m a}^{\dagger} \hat{B}_{m a}$, the population of another state is the expectation value of $\hat{B}_{n b}^{\dagger} \hat{B}_{n b}$; thus, the quartic terms represent the molecular energies in response to exciton populations of other molecules, which need not be resonant. For $\kappa \ll$ 1 , the only important coupling is the quartic term (this is the "weak coupling" regime in NMR spectroscopy), whereas in the case of resonant states both quardratic and quartic terms contribute ("strong coupling" regime) and the relative role of the former decreases with $\kappa$. Thus, clear signatures of quartic coupling should be expected, particulary for weakly coupled $(\kappa \ll 1)$ off resonant chromophores.

\section{Pump-Probe Signal for Aggregates of Two-Level Chromophores}

Our simulations were performed on an aggregate made of interacting two-level chromophores. We therefore omit the indices $a, b$, and $c$, which are redundant for two-level systems. Neglecting all nonresonant terms and only retaining the terms that conserve the number of excitons (Heitler-London approximation) reduces eq 3 to

$$
\hat{H}_{\mathrm{el}}=\sum_{m} \Omega_{m} \hat{B}_{m}^{\dagger} \hat{B}_{m}+\sum_{m n}^{m \neq n}\left(J_{m, n} \hat{B}_{m}^{\dagger} \hat{B}_{n}+K_{m, n} \hat{B}_{m}^{\dagger} \hat{B}_{n}^{\dagger} \hat{B}_{m} \hat{B}_{n}\right)
$$

where $\Omega_{m}$ is the excitation energy of chromophore $m, J_{m, n}$ is the resonant exciton coupling, and $K_{m, n}$ is the quartic coupling coefficient.

To account for relaxation processes, we added a linear coupling to a harmonic phonon bath. ${ }^{28}$ In general, all elements of the Hamiltonian can fluctuate. However, we only include fluctuations of the quadratic terms. This model describes dephasing as well as exciton migration. Each fluctuation is represented by a one-dimensional Brownian oscillator model, and the system-bath Hamiltonian is given by ${ }^{29}$

$$
\hat{H}_{\mathrm{SB}} \equiv \sum_{m n} \bar{h}_{m, n} q_{m n}(t) \hat{B}_{m}^{\dagger} \hat{B}_{n}
$$

where $\bar{h}_{m, n}$ is the coupling strength (in energy units) and $q_{m n}(t)$ is a dimensionless time-dependent bath coordinate. The relevant bath properties are contained in the spectral density:

$$
\bar{C}_{m n}^{\prime \prime}(\omega)=\frac{\bar{h}_{m n}^{2}}{2} \int_{-\infty}^{+\infty} \mathrm{d} t \exp (\mathrm{i} \omega t)\left\langle\left[q_{m n}, q_{m n}\right]\right\rangle
$$

We assume the overdamped Browninan oscillator model for the bath fluctuations with the spectral density:

$$
\bar{C}_{m n}^{\prime \prime}(\omega)=2 \pi \lambda_{m n} \frac{\Lambda_{m n} \omega}{\Lambda_{m n}{ }^{2}+\omega^{2}}
$$

where $\Lambda_{m n}$ defines the width of the spectral density (inverse relaxation time), $\lambda_{m n}=\bar{h}_{m n}{ }^{2} /\left(2 \Omega_{\text {Bath }}\right)$ is the coupling strength; $\Omega_{\text {Bath }}$ is the relevant bath frequency.

Only the optical field can change the number of excitations in our model. The interaction with the optical electric field, $\mathbf{E}(\mathbf{r}, t)$, is given by

$$
\hat{H}_{\mathrm{F}}=-\mathbf{E}(\mathbf{r}, t) \hat{\mathbf{P}}
$$

where $\hat{\mathbf{P}}$ is the dipole operator

$$
\hat{\mathbf{P}}=\sum_{m} \mu_{m}\left(\hat{B}_{m}^{\dagger}+\hat{B}_{m}\right)
$$

and $\mu$ is the transition dipole moment of the $m$ th chromophore.

The pump-probe signal was computed by solving the nonlinear exciton equations (NEE). ${ }^{11,28,30}$ The NEE consist of four types of coupled equations for the dynamical variables $B_{m}$ $=\left\langle\hat{B}_{m}\right\rangle, Y_{m, n}=\left\langle\hat{B}_{m} \hat{B}_{n}\right\rangle, N_{m, n}=\left\langle\hat{B}_{m}^{\dagger} \hat{B}_{n}\right\rangle$, and $Z_{m, n, k}=\left\langle\hat{B}_{m}^{\dagger} \hat{B}_{n} \hat{B}_{k}\right\rangle$. The Redfield relaxation operators of the NEE for the spectral density (eq 10) are given in ref 28. Numerical solution of these equations gives the polarization expressed by the expectation value of eq 13. In a pump-probe experiment the nonlinear polarization is generated by two optical pulses and the signal depends on the pump and probe frequencies $\left(\bar{\omega}_{1}\right.$ and $\bar{\omega}_{2}$, respectively) and their delay time $t_{\mathrm{d}}$. The pump-probe signal is defined as the difference between the absorption spectrum without and with the pump pulse. We denote the pump field as $\mathbf{E}_{1}(\mathbf{r}, t)$, and the probe as $\mathbf{E}_{2}(\mathbf{r}, t)$. The probe interacts with the nonlinear polarization generated in the direction $\left(\mathbf{k}_{\mathrm{s}}=\mathbf{k}_{1}-\mathbf{k}_{1}\right.$ $+\mathbf{k}_{2}$ ) and its absorption is measured. We express the probe field in the form

$$
\mathbf{E}_{2}(\mathbf{r}, t)=\tilde{\mathbf{E}}_{2}\left(t-t_{\mathrm{d}}\right) \exp \left(\mathrm{i} \mathbf{k}_{2} \mathbf{r}-\mathrm{i} \bar{\omega}_{2} t\right)+\text { c.c. }
$$

where $\tilde{\mathbf{E}}_{2}\left(t-t_{\mathrm{d}}\right)$ is the slowly varying optical field envelope, $\mathbf{k}_{2}$ is its wavevector, $\bar{\omega}_{2}$ is its carrier frequency and $t_{\mathrm{d}}$ is the delay time between the pump and the probe.

The total energy dissipated (or gained) from the probe is

$$
I\left(\bar{\omega}_{2}, t_{\mathrm{d}}\right)=\int_{+\infty}^{-\infty} \mathrm{d} t \int_{V} \mathrm{~d} \mathbf{r} \dot{\mathbf{E}}_{2}(\mathbf{r}, t) \cdot\langle\hat{\boldsymbol{P}}\rangle
$$

where $\dot{\mathbf{E}}_{2}(\mathbf{r}, t)=\left(\partial \mathbf{E}_{2}(\mathbf{r}, t)\right) /(\partial t)$ and $\langle\hat{\mathbf{P}}\rangle$ is the total polarization.

The interaction with the pump and probe generates a nonlinear polarization with all possible wavevectors $u \mathbf{k}_{1}+v \mathbf{k}_{2}$, where $u$ and $v$ are integers. We focus on the $\mathbf{k}_{2}$ component $(u=0$ and $v=1$ ), which determines the absorption of the probe. The numerical procedure of selecting a desired component of the 
total polarization in the NEE was given in ref 28 and the thirdorder polarization in the direction of probe is obtained by keeping the field sufficiently weak and subtracting the linear polarization from the total.

We express the $\mathbf{k}_{2}$ component of the third-order polarization $\mathbf{P}_{k_{2}}^{(3)}$ using its slowly varying amplitude:

$$
\mathbf{P}_{k_{2}}^{(3)}(\mathbf{r}, t)=\tilde{\mathbf{P}}_{k_{2}}^{(3)}(t) \exp \left(\mathrm{i} \boldsymbol{k}_{2} \mathbf{r}-\mathrm{i} \bar{\omega}_{2} t\right)+c . c .
$$

which is given by the NEE variables:

$$
\tilde{\boldsymbol{P}}_{k_{2}}^{(3)}(t)=\sum_{m} \mu_{m}\left(B_{m}^{(3)}\left(\mathbf{k}_{2}\right)+B_{m}^{(3)}\left(-\mathbf{k}_{2}\right)^{*}\right)
$$

where all variables $B_{m}^{(3)}\left(\mathbf{k}_{2}\right)=B_{m}\left(\mathbf{k}_{2}\right)-B_{m}^{(\mathrm{L})}\left(\mathbf{k}_{2}\right)$ are slowly varying functions within the RWA (rotating wave approximation); $B_{m}^{(\mathrm{L})}\left(\mathbf{k}_{2}\right)$ is the linear solution of the NEE obtained by neglecting all the nonlinear terms in NEE.

Using the slowly varying amplitude approximation for the optical field, the pump-probe spectrum is finally given by

$$
\begin{array}{r}
I\left(\bar{\omega}_{2}, t_{\mathrm{d}}\right)=-\bar{\omega}_{2} \operatorname{Im} \int_{-\infty}^{+\infty} \mathrm{d} t \sum_{m}\left[E_{m}^{*}\left(t-t_{\mathrm{d}}\right) B_{m}^{(3)}\left(\mathbf{k}_{2}\right)-\right. \\
\left.E_{m}\left(t-t_{\mathrm{d}}\right) B_{m}^{(3)}\left(-\mathbf{k}_{2}\right)^{*}\right]
\end{array}
$$

where $E_{m}\left(t-t_{\mathrm{d}}\right)=\mu_{m} \tilde{\mathbf{E}}_{2}\left(t-t_{\mathrm{d}}\right)$. Equation 18 was used in the present simulations.

\section{Numerical Simulations for the Photosynthetic Antenna LH2}

The light harvesting (LH) complex consists of coupled rings of chromophores which are chlorophylls (Chl) in green plants and bacteriochlorophyll (BChl) in the photosynthetic bacteria. ${ }^{31}$ The LH complexes absorb the sunlight and transfer the energy to the reaction center where it is converted into chemical energy. The recent determination of the structures of light-harvesting complexes in purple bacteria have extended the knowledge of functionality of a relatively simple photosynthetic system. ${ }^{32,33}$ The peripheral LH complex of purple bacteria (LH2) consists of two rings of BChls distinguished by their binding sites and separated by $20 \AA$ (see Figure 1). They are also distinguishable by their absorption spectrum: the BChls in the ring B800 absorb at $800 \mathrm{~nm}$ and the $\mathrm{B} 850$ ring absorbs at $850 \mathrm{~nm} .{ }^{31}$ The B850 excitations are red-shifted and delocalized due to intermolecular interactions (on the order of $600 \mathrm{~cm}^{-1}$ ). Due to larger distances between chromophores in the B800 ring, the interactions in B800 are weaker. The carotenoid (Car) molecules which absorb at $500 \mathrm{~nm}$ are found in close proximity to the BChls rings. The energy structure of $\mathrm{LH} 2$ allows to collect photons over a broad spectral range and to funnel the excitation energy $(\mathrm{Car} \rightarrow \mathrm{B} 800 \rightarrow$ B850) into the LH1 complex within 1 ps, which subsequently within $20-30 \mathrm{ps}$, transfers the energy to the reaction center. ${ }^{34-38}$

Recent experiments have suggested that the Car molecules, which form a separate exciton band from the BChls are sensitive to the state of BChls; their absorption band shifts when BChls are excited. ${ }^{20}$ Pump-probe experiments show that B800 excitation induces spectral changes in the Car band, whose decay time correlates well with the $\mathrm{B} 800 \rightarrow \mathrm{B} 850$ energy transfer, implying that the Car absorption depends on the populations of the B800 and $\mathrm{B} 850$ rings. Because this shift cannot be described by the standard exciton Hamiltonian (eq 1), additional local field effects were introduced phenomenologically on the basis of observa-

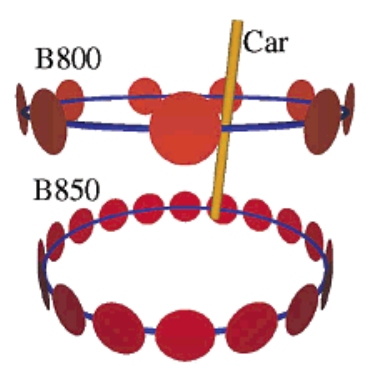

a)

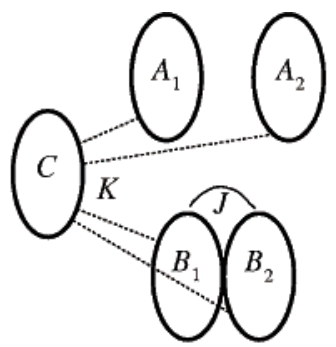

b)

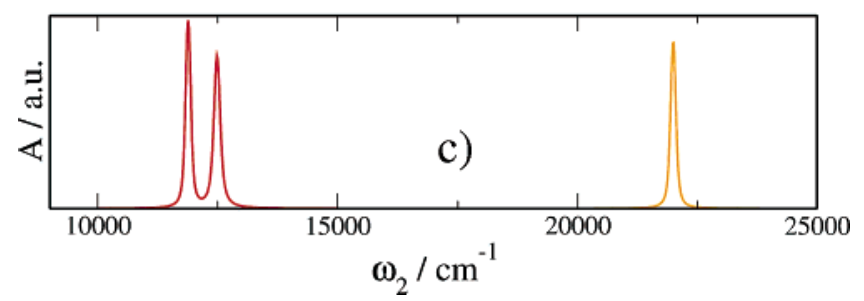

Figure 1. (a) Structure of light harvesting complex LH2: (red disks) BChls; (orange bar) Car. There are more Car molecules in the LH2, though we show one for clarity. (b) Our model consisting of five twolevel chromophores is shown on the right. $J$ represents quadratic coupling, and $K$, quartic. (c) The linear absorption spectrum.

tions that the carotenoid absorption bands are sensitive to electric fields created by the photoexcited chlorophylls. ${ }^{39-41}$

We have calculated the pump-probe signal of a simplified five-chromophore model system, which mimics the spectrum of the $\mathrm{LH} 2$ complex. The $\mathrm{B} 800$ and $\mathrm{B} 850$ rings were represented as two dimers $\mathrm{A}$ and $\mathrm{B}$ of two-level chromophores (see Figure 1b). Car is represented as an additional (C) two-level chromophore. We added quartic couplings between $\mathrm{A}-\mathrm{C}$ and $\mathrm{B}-\mathrm{C}$. Thus, in the Hamiltonian (eq 8) $m, n, \ldots$ now correspond to chromophores $\mathrm{A}_{1}, \mathrm{~A}_{2}, \mathrm{~B}_{1}, \mathrm{~B}_{2}$, and $\mathrm{C}$.

The following parameters were used. The energies of the chromophores of the $\mathrm{A}$ and $\mathrm{B}$ dimers are identical and correspond to excitation energies of isolated BChls: $\Omega_{\mathrm{A}_{1}}=\Omega_{\mathrm{A}_{2}}$ $=\Omega_{\mathrm{B}_{1}}=\Omega_{\mathrm{B}_{2}}=12500 \mathrm{~cm}^{-1}$. The quadratic coupling between chromophores $\mathrm{B}_{1}$ and $\mathrm{B}_{2}$ reflects couplings inside the $\mathrm{B} 850$ ring: $J_{\mathrm{B}_{1}, \mathrm{~B}_{2}}=600 \mathrm{~cm}^{-1}$. The excited-state energy of $\mathrm{C}$ chromophore represents Car absorption: $\Omega_{\mathrm{C}}=22000 \mathrm{~cm}^{-1}$. We also added weak coupling between $\mathrm{A}$ and $\mathrm{C}$ and between $\mathrm{B}$ and $\mathrm{C}: J_{\mathrm{A}_{1}, \mathrm{C}}=J_{\mathrm{A}_{2}, \mathrm{C}}=J_{\mathrm{B}_{1}, \mathrm{C}}=J_{\mathrm{B}_{2}, \mathrm{C}}=50 \mathrm{~cm}^{-1}$. The coupling strength was adjusted to preserve the excitonic band structure inside B dimer. To reproduce the large Car line shifts observed when B800 are populated and the smaller shifts when B850 rings are populated, ${ }^{20}$ we assumed different quartic couplings between $\mathrm{A}-\mathrm{C}$ and $\mathrm{B}-\mathrm{C}$ : $U_{\mathrm{A}_{1}, \mathrm{C}}=U_{\mathrm{A}_{2}, \mathrm{C}}=300 \mathrm{~cm}^{-1}$ and $U_{\mathrm{B}_{1}, \mathrm{C}}$ $=U_{\mathrm{B}_{2}, \mathrm{C}}=100 \mathrm{~cm}^{-1}$.

Exciton relaxation was incorporated by adding diagonal and off-diagonal couplings with the bath. Diagonal couplings lead to spectral line broadening, whereas off-diagonal couplings cause redistribution of populations. To reproduce the typical broadenings and population redistribution rates, we used $\lambda_{m, m}=1.5$ $\mathrm{cm}^{-1}$ and $\lambda_{m, n}=0.66 \mathrm{~cm}^{-1}$. The bath spectral width was $\Lambda_{m, n}$ $=\Lambda_{m, m}=100 \mathrm{~cm}^{-1}$ and the temperature $k_{\mathrm{B}} T=200 \mathrm{~cm}^{-1}$. Dipole moments of all transitions were taken to be identical and parallel to the optical field. The transition dipoles of the dimers inside A and B are antiparallel.

The pump-probe signal was simulated using Gaussian optical pulses with variance $\sigma_{0}=100 \mathrm{fs}$. The pump frequency was fixed at the $\mathrm{B} 800$ absorption $\bar{\omega}_{1}=12500 \mathrm{~cm}^{-1}$ and the probe frequency, $\bar{\omega}_{2}$, was tuned in the ranges $11000-14000$ and $21000-23000 \mathrm{~cm}^{-1}$. The time delay between the pump and 

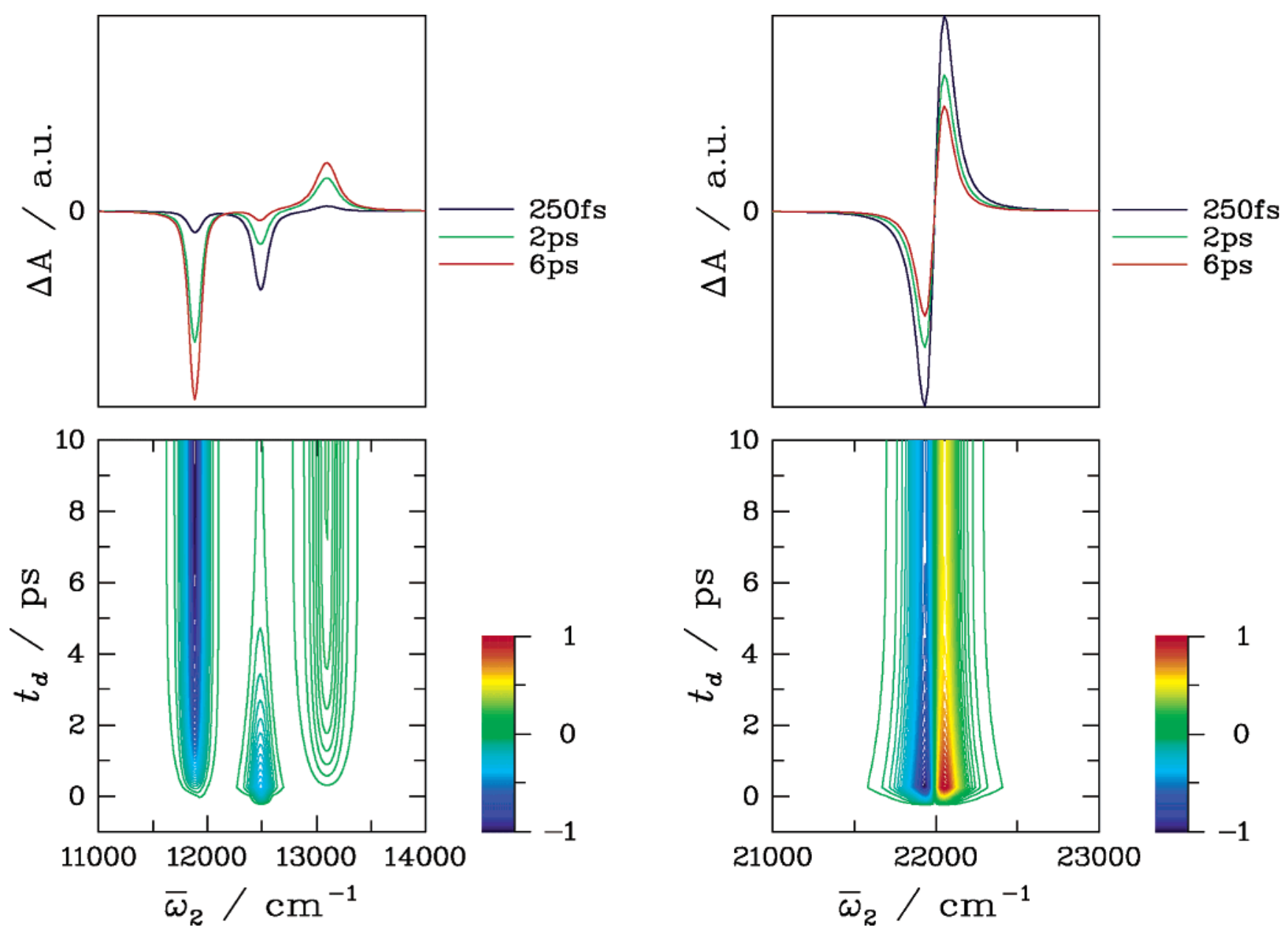

Figure 2. Lower panel: simulated pump-probe spectrum in the region $11000-14000 \mathrm{~cm}^{-1}$. The pump pulse excites A chromophores $(12500$ $\mathrm{cm}^{-1}$ ). Upper panel: slices of the pump-probe spectrum at different delay times.

probe was varied between -2 to +20 ps. The step size of the NEE integration was 2 fs. The pulse amplitudes were kept low to stay within the third-order response regime.

The simulated linear absorption in the regions of $\mathrm{BCls}$ and Car presented in Figure 1c consists of three bands: $11900 \mathrm{~cm}^{-1}$ corresponds to $\mathrm{B} 850,12500 \mathrm{~cm}^{-1}$ corresponds to $\mathrm{B} 800$, and $22000 \mathrm{~cm}^{-1}$ corresponds the Car absorption (the exciton state at $13100 \mathrm{~cm}^{-1}$ is optically forbidden), with line widths (fwhm) $100 \mathrm{~cm}^{-1}$. Figures 2 and 3 show the simulated pump-probe spectrum, where the pump excites the A dimer at $12500 \mathrm{~cm}^{-1}$ and probing was done either in the regions $11000-14000 \mathrm{~cm}^{-1}$ (chlorophylls) or $21000-23000 \mathrm{~cm}^{-1}$ (carotenoids). The exciton population dynamics is presented in Figure 4a. The excitation of A is transferred to B within a few picoseconds, mimicing the experimental observations of $\mathrm{B} 800 \rightarrow \mathrm{B} 850$ population transfer. The pump-probe spectrum in the B850 region (Figure 2) also reveals energy flow $\mathrm{A} \rightarrow \mathrm{B}$ and later excitonic effects of the $\mathrm{B}$ dimer (induced absorption into twoexciton state at $13100 \mathrm{~cm}^{-1}$ ). The $\mathrm{C}$ absorption band region depicted in Figure 3 shows shifts of $\mathrm{C}$ absorption to higher energies immediately after the excitation. The amplitude of the opposite peaks at 21950 and $22050 \mathrm{~cm}^{-1}$ decreases as population is transferred to the $\mathrm{B}$ dimer and remains constant after the exciton transfer is completed. This dynamics of peak amplitudes is shown in Figure 4b. The pump-probe spectrum in the $\mathrm{C}$ region (Figure 3 ) is solely induced by the quartic

Figure 3. Lower panel: simulated pump - probe spectrum in the region $21000-23000 \mathrm{~cm}^{-1}$. The pump pulse excites A chromophores (12 500 $\mathrm{cm}^{-1}$ ). Upper panel: slices of the pump-probe spectrum at different delay times.
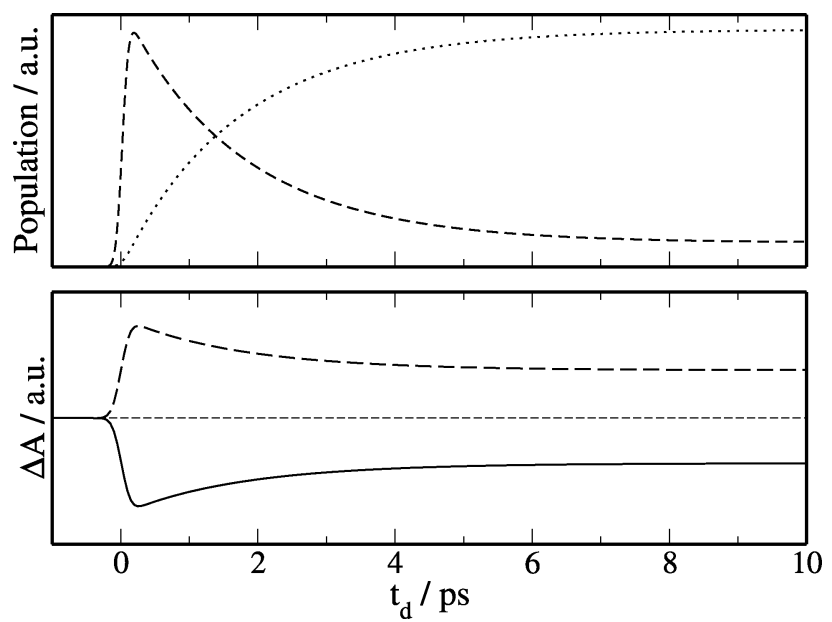

Figure 4. Time evolution of populations and pump-probe spectra during the experiment. The population of A (B) chromophores (upper panel) is shown with dashed (dotted) lines. The peak amplitude (lower panel) at $21950 \mathrm{~cm}^{-1}$ is shown with a solid line and at $22050 \mathrm{~cm}^{-1}$ with a dashed line.

coupling $\left(K_{m n}\right)$ and disappears when $K_{m n}$ is switched off. In contrast, the B spectra (Figure 2) are unaffected by the quartic couplings.

It should be noted that quartic couplings between off resonant optical transitions of different pigments $(\mathrm{Qx}$ and Soret bands of $\mathrm{BChl}$, states of Car) should also affect the pump-probe 
spectrum. We assumed a simple model to illustrate the effect of quartic couplings and the value of $100 \mathrm{~cm}^{-1}$ used in our simulations should be considered as a rough estimate of the effect in LH2. More detailed simulations that include all pigments of B800 and B850, and Car molecules will be required for a quantitative comparison with experiment. The population redistribution as well as intramolecular vibrational relaxation (which is important in LH2) need to be considered as well.

\section{Discussion}

Molecular aggregates are commonly described by an exciton Hamiltonian that conserves the number of excitations. Nonconserving processes are controlled by the ratio of the intermolecular coupling to the optical frequency $(J / \Omega)$, which is typically small and may be neglected. This provides a convenient computational scheme as well as a basis for an intuitive physical picture, because the energy spectrum consists of well-separated groups of energy-levels representing single and double excitations etc. ${ }^{42}$ Because only the radiation field can change the number of excitations, we can design nonlinear techniques to probe specific electronic excited states. The lowest (one-exciton) manifold is accessible by linear optical techniques (e.g., linear absorption) whereas the two times excited aggregates (twoexciton states) can be monitored by third-order spectroscopies. ${ }^{42}$ Successively higher manifolds can be probed with higher order techniques.

The lowest order term in the Hamiltonian that conserves the number of excitations is the quadratic coupling. It creates exciton states that are observed in linear absorption as new spectral lines not seen in the isolated molecules. The higher order terms that also conserve the number of excitations are quartic. The linear absorption is not sensitive to the quartic couplings that describe couplings among several excitations. Nonlinear techniques such as pump-probe monitor spectral line shifts of particular states induced by population redistribution in the other states. Our simulated pump-probe spectrum shows that the time-dependent interband line shift is directly attributed to the quartic coupling between excitons. The quartic coupling enters the NEE as a new term $\left(K_{m n}+K_{n m}\right)\left\langle\hat{B}_{n}^{\dagger} \hat{B}_{n} \hat{B}_{m}\right\rangle$ in the equation for variable $B_{m}$. Correspondingly, the magnitude of the absorption line shift of state $m$ is roughly equal to $2 K_{m n} \rho_{n n}$, where $\rho_{n n}$ is the population of the state $n$. In the regime of field intensities used in our simulations the populations of the states are very low. Therefore, the line shift is negligible and the $100 \mathrm{~cm}^{-1}$ separation of the positive and negative peaks in the pumpprobe spectrum (which is a result of the difference of two Lorentzian functions) in the Car region originates from the absorption linewidth. For strong saturating laser fields, the shift of the absorption line could be on the order of $K_{m n}\left(\rho_{n n}\right.$ can be as large as $1 / 2$ ) and separation of two Lorentzians could be observed when $K_{m n}$ is comparable to the line width. The NEE only require the one-exciton block of the Hamiltonian to calculate the response. The sum over states approaches ${ }^{42}$ in contrast, require diagonalizing the complete Hamiltonian and finding one- and two-exciton states. The quartic coupling enters the diagonal of two-exciton block by shifting the combination state energies, resulting in a spectral shift of the induced absorption and stimulated emission.

The spectral shifts are usually associated with the local electric field acting on the molecule (Stark effect). This is a consequence of populations of electronic states and of solvation effects, i.e., relaxation processes in the environment. Our simulations demonstrate that the line shift in pump-probe spectroscopy can be attributed to quartic coupling. This can be interpreted as a local field effect; $K_{m n}$ represents the difference of the energy of chromophore $m$ caused by the electric field generated by the charge distribution of an adjacent chromophores $n$. Solvation effects that are not included in our Hamiltonian may contribute to the shift as well.

Quartic couplings are commonly used in multidimensional NMR and were recently used in nonlinear vibrational spectroscopy to describe coupled localized vibrational motions of peptides. ${ }^{26}$ The Hamiltonian of these systems is obtained by expanding the potential energy surface of molecule in the powers of nuclear coordinates. Thus quadratic, cubic, and quartic coupling coefficients appear as second-, third-, and fourth-order derivatives of anharmonic potential energy surface. Quartic couplings should show clear signatures in other third-order spectroscopies of electronic and vibrational excitons.

Acknowledgment. This material is based upon work supported by the National Science Foundation grant no. CHE0132571. The support of the National Institutes of Health grant no. 1 RO1 GM59230-10A2 is gratefully acknowledged. We dedicate the article to Gerry Small whose pioneering work on molecular excitons has been an invaluable source of inspiration.

\section{References and Notes}

(1) Mukamel, S. Аnпи. Rev. Phys. Chem. 2000, 51, 691.

(2) Ernst, R. R.; Bodenhausen, G.; Wokaun, A. Principles of Nuclear Magnetic Resonance in One and Two Dimensions; Clarendon Press: Oxford, U.K., 1987. Sanders, J. K. M.; Hunter, B. K. Modern NMR Spectroscopy-a Guide for Chemists. Second Edition; Oxford University Press: Oxford, New York, 1993.

(3) Davydov, A. S. Theory of Molecular Excitons; McGraw-Hill: New York, 1962

(4) Silinsh, E. A.; Capek, V. Organic Molecular Crystals: Interaction, Localization and Transport Phenomena; AIP Press: New York, 1994.

(5) Juzeliunas, G.; Knoester, J. J. Chem. Phys. 2000, 112, 2325.

(6) Kobayashi, T. J-aggregates; World Scientific: Singapore, 1996.

(7) Mukamel, S.; Chemla, D. S. Special Issue on Confined Excitations in Molecular and Semiconductor Nanostructures. Chem. Phys. 1996, 210

(8) Forrest, S. R. Chem. Rev. 1997, 97, 1793.

(9) McBranch, D. W.; Sinclair, M. B. The nature of the photoexcitations in conjugated polymers; Sariciftci, World Scientific Publihsing: Singapore, 1997.

(10) Baldo, M. A.; Thompson, M. E.; Forrest, S. R. Nature 2000, 403, 750.

(11) Chernyak, V.; Zhang, W. M.; Mukamel, S. J. Chem. Phys. 1998, $109,9587$.

(12) Bradforth, S. E.; Jimenez, R.; van Mourik, F.; van Grondelle, R.; Fleming, G. R. J. Phys. Chem. 1995, 99, 16179. Jimenez, R.; Dikshit, S. N.; Bradforth, S. E.; Fleming, G. R. J. Phys. Chem. 1996, 100, 6825.

(13) Joo, T.; Albrecht, A. C. Chem. Phys. 1993, 176, 233.

(14) Joo, T.; Jia, Y. W.; Yu, J. Y.; Jonas, D. M.; Fleming, G. R. J. Phys. Chem. 1996, 100, 2399.

(15) Jimenez, R.; van Mourik, F.; Yu, J. Y.; Fleming, G. R. J. Phys. Chem. B 1997, 101, 7350.

(16) Yu, J. Y.; Nagasawa, Y.; van Grondelle, R.; Fleming, G. R. Chem. Phys. Lett. 1997, 280, 404.

(17) Reddy, N. R. S.; Cogdell, R. J.; Zhao, L.; Small, G. J. Photochem. Photobiol. 1993, 57, 35.

(18) Caro, C. D.; Visschers, R. W.; van Grondelle, R.; Völker, S. J. Phys. Chem. 1994, 98, 10584.

(19) van der Laan, H.; Schmidt, T.; Visschers, R. W.; Visschers, K. J.; van Grondelle, R.; S. Völker, S. Chem. Phys. Lett. 1990, 170, 231.

(20) Herek, J. L.; Polivka, T.; Pullerits, T.; Fowler, G. J. S.; Hunter, C. N.; Sundstrom, V. Biochemistry 1998, 37, 7057.

(21) Abramavicius, D.; Gulbinas, V.; Valkunas, L.; Shiu, Y.-J.; Liang, K. K.; Hayashi, M.; Lin, S. H. J. Phys. Chem. A 2002, 106, 8864.

(22) Chachisvilis, M.; Zewail, A. H. J. Phys. Chem. A 1999, 103, 7408

(23) Zewail, A. H. Femtochemistry - Ultrafast Dynamics of The Chemical Bond Vol. I and II; 20th Century Chemistry Series; World Scientific: Singapore, 1994.

(24) Pullerits, T.; Chachisvilis, M.; Sundström, V. J. Phys. Chem. 1996, 100, 10787. Pullerits, T.; Chachisvilis, M.; Jones, M. R.; Hunter, C. N.; Sundström, V. Chem. Phys. Lett. 1994, 224, 355.

(25) Zhang, W. M.; Meier, T.; Chernyak, V.; Mukamel, S. J. Chem. Phys. 1998, 108, 7763. Zhang, W. M.; Meier, T.; Chernyak, V.; Mukamel, S. Philos. Trans. R. Soc. London, Ser. A 1998, 356, 405.

(26) Scheurer, C.; Mukamel, S. J. Chem. Phys. 2001, 115, 4898. Scheurer, C.; Mukamel, S. J. Chem. Phys. 2002, 116, 6803. Scheurer, C.; Mukamel, S. Bull. Chem. Soc. Jpn. 2002, 75, 989. 
(27) Mukamel, S.; Berman, O. J. Chem. Phys. 2003, 119, 12194.

(28) Mukamel, S.; Abramavicius, D. Chem. Rev. 2004, in press.

(29) Cherniak, V.; Mukamel, S. J. Chem. Phys. 1996, 105, 4565.

(30) Zhang, W. M.; Chernyak, V.; Mukamel, S. J. Chem. Phys. 1999, $110,5011$.

(31) van Amerogen, H.; Valkunas, L.; van Grondelle, R. Photosynthetic Excitons; World Scientific: Singapore, 2000.

(32) Freer, A.; Prince, S.; Sauer, K.; Papiz, M.; HawthornthwaiteLawless, A.; McDermott, G.; Cogdell, R.; Isaac, N. W. Structure 1996, 4 449.

(33) Koepke, J.; Hu, X.; Muenke, C.; Schulten, K.; Michel, H. Structure 1996, 4, 581 .

(34) Koyama, Y.; Kuki, M.; Andersson, P. O.; Gillbro, T. Photochem. Photobiol. 1996, 63, 243.

(35) Ricci, M.; Bradforth, S. E.; Jimenez, R.; Fleming, G. R. Chem. Phys. Lett. 1996, 259, 381
(36) Monshouwer, R.; Ortiz de Zarate, I.; van Mourik, F.; van Grondelle, R. Chem. Phys. Lett. 1995, 246, 341.

(37) Pullerits, T.; Hess, S.; Herek, J. L.; Sundström, V. J. Phys. Chem. $B$ 1997, 101, 10560 .

(38) Zazubovich, V.; Jankowiak, R.; Riley, K.; Picorel, R.; Seibert, M.; Small, G. J. J. Phys. Chem. B 2003, 107, 2862. Zazubovich, V.; Jankowiak, R.; Small, G. J. J. Luminescence 2002, 98, 123.

(39) Crielaard, W.; Wissechers, R. W.; Fowler, G. J. S.; van Grondelle, R.; Hellingwerf, K. J.; Hunter, C. N. Biochim. Biophys. Acta 1994, 1183 473.

(40) Gottfried, D. S.; Steffen, M. A.; Boxer, S. G. Science 1991, 251, 662

(41) Gottfried, D. S.; Steffen, M. A.; Boxer, S. G. Biochim. Biophys Acta 1991, 1059, 76.

(42) Mukamel, S. Principles of Nonlinear Optical Spectrscopy; Oxford University Press: Oxford, New York, 1995. 